A.-S. Biesalski ${ }^{1}$, L. Peters ${ }^{2}$, L. Tönges ${ }^{1}$, S. Klebe ${ }^{2}$, F. Fincke ${ }^{3}$, K. Bornkamm ${ }^{4}$, J. Brich ${ }^{4}$, F. Schmidt-Graf ${ }^{5}$

${ }^{1}$ Neurologische Klinik St. Josef Hospital Bochum, Ruhr-Universität Bochum, Bochum, Deutschland

${ }^{2}$ Klinik für Neurologie, Universitätsklinikum Essen, Essen, Deutschland

${ }^{3}$ Klinik für neurologische Intensivmedizin und neurologische Frührehabilitation, Evangelisches Krankenhaus Oldenburg, Oldenburg, Deutschland

${ }^{4}$ Klinik für Neurologie und Neurophysiologie, Universitätsklinikum Freiburg, Freiburg, Deutschland

${ }^{5}$ Klinik und Poliklinik für Neurologie, Technische Universität München, Klinikum rechts der Isar, München, Deutschland

\title{
Die Auswirkungen der Pandemie auf die neurologische Lehre: Not macht erfinderisch!
}

Die Coronapandemie ist in alle Lebens- und Kulturbereiche vorgedrungen und hat auch den Wissenschafts- und Lehrbetrieb der medizinischen Fakultäten zu erheblichen Anpassungen gezwungen. Von Lehrenden in der Neurologie wurde überwiegend berichtet, dass ein Großteil der praktischen Lehre (wie Lehrvisiten, praktische Kurse, Hospitationen, Seminare und Kleingruppenunterricht) ersatzlos entfiel, während die Vorlesungen im digitalen Format stattfinden konnten [1]. Neben der erheblichen Mehrbelastung für Lehrbeauftragte und -koordinator ${ }^{\star}$ innen litten auch Studierende unter dem Ausfall der Präsenzlehre [2].

Inzwischen, fast 2 Jahre nach der ersten Welle, scheint sich an den Universitätskliniken und Lehrkrankenhäusern eine Art „Zwischenregelbetrieb“ einzustellen. Unterrichtsformate, die im vergangenen Jahr noch aus der Not heraus geboren wurden, um eine kurze Zeit lang die Lehre aufrechtzuerhalten, konnten in den vergangenen 3 Semestern erprobt und verbessert werden.

Wir stellen Ihnen 5 Lösungen vor, die an den Fakultäten in Bochum, Oldenburg, Freiburg, München und Essen zur Lehre in der Neurologie entwickelt wurden:

An der Ruhr-Universität Bochum wurde die Hauptvorlesung im 6. und 7. Semester durch eine asynchrone, digitale Vorlesung ersetzt. Da hierdurch der eigentliche Vorlesungszeitraum "frei“ wird, kann die ursprüngliche Vorlesungszeit nun für die Präsenzlehre genutzt werden. Nur so ist es möglich, den knapp 300 Studierenden an den 3 neurologischen Kliniken seit dem Wintersemester wieder einen Unterricht am Krankenbett (UaK) zu ermöglichen. Während in den 2 Semestern zuvor seit Beginn der Pandemie noch in kleineren Gruppen nacheinander im Wechsel - zunächst $45 \mathrm{~min} \mathrm{UaK}$, dann 45 min Fallvortrag mit jeweils 20 Studierenden - unterrichtet wurde, ist inzwischen fast eine Rückkehr zum Normalbetrieb möglich gewesen - unter strengen Hygieneauflagen, versteht sich. Organisatorisch ist der UaK eine Herausforderung, die über die reine neurologische Lehre weit hinausgeht. Doch die Studierenden sind

\section{Redaktion}

P. Berlit, Berlin

DGNeurologie $2022 \cdot 5(1): 5-8$

https://doi.org/10.1007/s42451-021-00405-8

Angenommen: 26. November 2021

Online publiziert: 17. Dezember 2021

๑) Springer Medizin Verlag GmbH, ein Teil von Springer Nature 2021 dankbar für die Möglichkeit des Unterrichts vor Ort - auch, wenn aufgrund der Situation manchmal kein/e Patient*in zur Verfügung steht, und die neurologische Untersuchung dann an Kommiliton*innen geübt wird.

Auch von Seiten der Lehrenden kommt positives Feedback: Man sei froh, die Studierenden wieder direkt unterrichten und auch Hilfestellungen geben zu können. Der Onlineunterricht sei keine Dauerlösung für die praktischen Inhalte. Ein digitales Untersuchungsskript sowie Videomaterial helfen seit dem vergangenen Semester beim Erlernen der praktischen Untersuchungsschritte.

Um den Hygienevorgaben gerecht werden zu können, erhalten alle Studierenden ausreichende Selbsttests im Vorfeld. Zusätzlich ist der Impfstatus auf dem Studierendenausweis vermerkt und wird konsequent kontrolliert.

Auch im Modellstudiengang an der Universität Oldenburg wurden die Vorlesungen vollständig in ein digitales Format gebracht, das von den Dozierenden in Form von digitalen Präsentationsmaterialien oder vorab aufgezeichneten Vorträgen bereitgestellt wird. Auch die Neurologieseminare finden rein digital statt, hier werden je nach Veranstaltungsfokus ganz unterschiedliche Konzepte verfolgt. Bei einigen Seminaren werden den Studierenden Materialien (oder z.B. Fallvignetten) zur Verfügung gestellt, Ausarbeitungen von Dozierenden beurteilt, und im Anschluss erhalten die Seminargruppen ein Feedback. Andere Seminare finden im Rahmen einer digitalen Konferenz statt.

Der Untersuchungskurs musste zwischen März und September 2020 komplett pausiert und von den Studierenden im Verlauf nachgeholt werden. Inzwischen können die Untersuchungskurse unter eingeschränkten Bedingungen unter Berücksichtigung der Hygieneauflagen wieder begrenzt stattfinden, wobei einzelne Untersuchungsschritte theoretisch bleiben. Insbesondere erhalten die Studierenden Lehrmaterialien wie einen Modulplan und Videos zur Übung zuhause. Die praktischen Übungen des Untersuchungskurses wurden zu Beginn des obligatorischen, 4-wöchigen Blockpraktikums nachgeholt. Die Umsetzung des Blockpraktikums konnte, unter Berücksichtigung der aktuellen Gegebenheiten und Auflagen, im Verlauf wieder aufgenommen werden. Eine Besonderheit des Modellstudiengangs sind in Oldenburg sicherlich die mündlich-praktischen Prüfungsformate wie OSCE und Mini-CEx (s. Infobox 1), die auf das klinische Blockpraktikum vorbereiten. Da ein OSCE aktuell nicht umsetzbar ist, wird die 


\section{Infobox 1}

OSCE steht für "objective structured clinical examination". Hierbei handelt es sich um ein praxisorientiertes Prüfungsdesign, das Studierende anhand eines Bewertungsbogens immer gleich (daher objektiv) beurteilt. In der Regel werden dabei "skills“ wie Anamnesegespräche, klinische Untersuchungen eines Organsystems oder ärztliche Tätigkeiten wie Blutabnehmen, Reanimieren, chirurgisches Nähen oder Legen von Zugängen abgefragt. In der OSCE-Prüfung durchlaufen die Studierenden einen Parcours aus mehreren Stationen und werden z. B. nach Ablauf einer Zeitfrist von $5 \mathrm{~min}$ per Zeichen zur nächsten Station aufgefordert. Pro Station gibt es meist eine/n Prüfer*in und eine/n Simulationspatient*in. Gemäß Masterplan 2020 sollen OSCE-Prüfungen zukünftig ein fester Bestandteil des Medizinstudiums werden und als Staatsexamensprüfungen genutzt werden.

Mini-CEx steht für "mini clinical examination" und gehört zu den arbeitsplatzbasierten Prüfungen. Das Format überprüft im klinischen Setting am/an der Patient*in eine Anzahl vordefinierter klinisch-praktischer Fertigkeiten, die in einem Praxisblock erlernt werden konnten. Zu diesem Repertoire gehören eine Reihe von universalen, ärztlichen Handgriffen und Abläufen, die jede/r angehende Ärzt*in beherrschen sollte. Neben den fachspezifische (Untersuchungs-)Techniken gehören auch das souveräne Auftreten in Interaktion mit dem/der Patient*in zum medizinischen "skill repertoire".

praktische Prüfung in Form eines Mini-CEx (mini clinical examination) im Rahmen des Blockpraktikums durchgeführt.

Erste Rückmeldungen der Studierenden in Oldenburg zeigen, dass die digitalen Lehrmethoden gut angenommen werden. Ob die Veränderungen einen langfristigen Mehrwert bringen, kann gegenwärtig noch nicht prognostiziert werden, jedoch wird das Vorhandensein digitaler Materialien wie Videos insgesamt als positiv empfunden.

Auch an der Klinik für Neurologie und Neurophysiologie der Universität Freiburg wurde die klinische Lehre im Blockpraktikum Neurologie in den "Coronasemestern“ zunächst komplett digital umgesetzt und dann schrittweise in ein Hybridformat umgestaltet. Dabei kommt eine Kombination aus asynchronen Formaten („screencasts“/Handouts von Seminaren/Vorlesungen, Videos zum Untersuchungskurs und zur Patientenvorstellung), synchronen Onlineveranstaltungen (Diskussions-/Fragestunden, Onlineseminare, „teambased learning" [3] via Zoom, Zoom Video Communications, San José, Kalifornien, Vereinigte Staaten) und einzelnen Präsenzveranstaltungen (Untersuchungskurs, Bedside-Kurs) zum Einsatz.

Die asynchronen Formate werden von den Studierenden aufgrund der flexiblen Zugriffsmöglichkeiten sehr geschätzt, die Qualität wird als analog zu den Präsenzveranstaltungen eingeschätzt. Insbesondere die Videos von Patientenvorstellungen, die einen klinischen Bezug herstellen sollten (z.B. ein interprofessionell [Ärzt*in, „Parkinson nurse“] geführtes Interview mit einem Parkinson-Patienten inkl. Untersuchung, wobei auch die psychosozialen Aspekte der Krankheit beleuchtet wurden), fanden großen Anklang bei den Studierenden.

Bei den synchronen Onlineveranstaltungen mittels Zoom zeigte sich zunächst meist das vielfach beschriebene Phänomen der „stummen schwarzen Kacheln“" mit wenig aktiver Mitarbeit der Studierenden. Erst mit Hilfe der auf die Gegebenheiten der Onlinelehre angepassten Methode des „team-based learning“ (Kleingruppendiskussionen mit der Zoom-Funktion „breakout-sessions“ innerhalb einer Videokonferenz) konnten lebhafte und interaktive Lehrveranstaltungen erreicht werden.

Die Planung und Durchführung von Präsenzveranstaltungen dagegen waren durch die dynamische Pandemielage mit rasch wechselnden Vorgaben erschwert, und eine hohe Flexibilität mit kurzfristiger Absage und Umplanung der Veranstaltungen war gefordert. Erst durch die Entwicklung einheitlicher Vorgaben des Studiendekanats hinsichtlich Organisation und Hygienevorschriften des Unterrichts am Krankenbett konnten der neurologische Untersuchungskurs und ein Hybrid-BedsideKurs wieder etabliert werden, bei dem die einzelnen Patientenvorstellungen mit jeweils wenigen Studierenden direkt aus dem Patientenzimmer per Videokonferenz in einen Raum mit der restlichen Gruppe der Studierenden übertragen wurden. Datenschutzvorgaben und die technische Umsetzung bleiben jedoch auch in Freiburg weiterhin herausfordernd.

In der Neurologie des Universitätsklinikums Essen konnten die Vorlesungen, die zumeist im Vorjahr aufgezeichnet worden waren und in digitaler Form vorlagen, den Studierenden über die Lehrplattform Moodle im Vorfeld zur Verfügung gestellt werden. $\mathrm{Zu}$ den im Stundenplan hinterlegten Vorlesungsterminen fanden dann, angelehnt an das Konzept des „flipped classroom“, Webinare mittels Zoom mit den entsprechenden Dozierenden statt, die nun auf das Vorwissen der Studierenden aufbauen und in z. T. interaktiven Fallbesprechungen und Patientenvorstellungen sowie einem Einblick in die neurophysiologische Diagnostik das Fachgebiet der Neurologie „erlebbar“ machen konnten.

Die besondere Herausforderung bestand darin, eine adäquate Lösung für das neurologische Praktikum zu finden, das durch die geltenden Infektionsschutzbestimmungen nicht in Präsenz stattfinden konnte. Um eine Interaktion mit den Patient ${ }^{\star}$ innen $\mathrm{zu}$ ermöglichen, fand ein live per Zoom übertragenes „bedside-teaching“ mit thematischen Schwerpunkten statt. Hierbei wurden die Teilnehmenden ausführlich über den Ablauf, aber auch über die Limitationen in Hinblick auf die Datensicherheit aufgeklärt und eine Einverständniserklärung eingeholt. Weitere Maßnahmen zur Erhöhung der Datensicherheit, aber auch zur Durchführung mit Erläuterung von Optimierungsschritten können in der Arbeit von Oster et al. [4] nachvollzogen werden. Die Studierenden konnten durch dieses live übertragene Format selbstständig die strukturierte neurologische Anamnese erheben, gemeinsam mit den Dozierenden sinnvolle klinische Untersuchungsschritte besprechen, die dann vor der Kamera gezeigt und zum praktischen Nachvollziehen im privaten Umfeld entsprechend erklärt wurden. Im Nachgang fand dann eine oberärztlich geführte Aufarbeitung des Falls mit einer Erläuterung der Befunde und Besprechung der weiteren diagnostischen und therapeutischen Schritte statt. Begleitende Evaluationen ergaben eine hohe Akzeptanz und Zufriedenheit bei den Studierenden.

In der Neurologischen Klinik der TU München war ein Vorteil zu Beginn der Pandemie, dass es bereits ein „flipped classroom model“ der neurologischen Hauptvorlesung gab. So konnten vorhandene Materialien und Onlinetools übernommen und an die Pandemiebedingungen angepasst werden: In einem Moodle-Kurs waren vor jeder Vorlesung ein "factsheet“, ein kurzer Film und 2 MC-Fragen zu bearbeiten, die Vorlesung fand als Zoom-Veranstaltung statt. Nach anfänglich we- 
Hier steht eine Anzeige.

Springer 
niger Interaktion bei auch meist ausgeschalteter Kamera gewöhnten sich nach und nach alle an Onlineveranstaltungen, und es gab zunehmende Diskussionen sowohl mündlich als auch im Chatverlauf, sodass hier kein Nachteil mehr zu den Zeiten vor der Pandemie zu bemerken war. Die Teilnahme an der Vorlesung hat sich durch das Onlineangebot sogar deutlich erhöht. Die Abschlussklausur konnte jeweils durch Ausweichen auf mehrere Hörsäle mit entsprechendem Abstand bei erhöhtem Personalaufwand durchgeführt werden. Bedside-Kurs und Blockpraktikum fanden im SS 2020 auf freiwilliger Basis mit Bereitstellung von 2 „Screencasts“ zur neurologischen Untersuchung sowie der Möglichkeit zu einer Zoom-Veranstaltung statt. Seit Oktober 2020 fanden die praktischen Kurse nun in halber Gruppengröße und dadurch nur an 1 statt 2 Tagen vor Ort mit Patienten statt. Auch in München führten die Studierenden vorab einen COVID-19-Selbsttest durch. Inzwischen werden seit dem laufenden Wintersemester die praktischen Kurse wieder in normalem Umfang mit voller Gruppengröße durchgeführt, mit 3G-Regel.

Erfreulicherweise hat sich in München sowohl für Studierende als auch für Lehrende gezeigt, dass die Halbierung der Teilnehmerzahl auch zu einer Intensivierung des Lernens führt, und dadurch der Nachteil einer reduzierten Stundenzahl wieder in großen Teilen ausgeglichen werden kann. Im Bedside-Kurs wird seit dem WS 2020 im Rahmen eines Innovationsprojektes mit wissenschaftlicher Begleitung untersucht, inwieweit über ein „flipped-classroom model“ bereits zuhause Wissen vermittelt werden und die Präsenzzeit dann besser und intensiver in der Klinik mit Dozierenden und Patienten genutzt werden kann. Zwei freiwillige Lehrveranstaltungen in der Neurologie („case discussion round“ und Differenzialdiagnoseseminar) werden jeweils online angeboten, was bei kleiner Gruppengröße mit bis zu 20 Studierenden relativ gut umsetzbar erscheint. Die Diskussionsbeteiligung und damit meist die Qualität der Veranstaltungen stellen sich sehr heterogen dar und hängen in erster Linie von den Teilnehmenden ab.

Zusammengefasst lassen sich Lehrveranstaltungen, die Fakten-, Handlungs- und Begründungswissen vermitteln, gut als digitale Formate umsetzen. Das Herzstück der neurologischen Lehre jedoch, der Unterricht am Patientenbett mit fokussierter Anamnese und neurologisch-topischer Diagnostik, der praktische Fertigkeiten und Handlungskompetenz vermittelt, kann nicht komplett digital ersetzt werden, sondern erfordert Präsenzformate. Ein Hybridformat kann dabei eine Übergangslösung sein, bis die Studierenden wieder ausführlicher im Patientenkontakt lernen können. Konzepte des „flipped classroom" bieten sich hier besonders an, wenn die Unterrichtseinheiten zugunsten der Hygienebedingungen verkürzt werden müssen

Letztlich hat die Pandemie für alle Lehrenden eine erhebliche Mehrbelastung bei der Unterrichtsplanung und -gestaltung mit sich gebracht. Die z. T. rasch wechselnden Hygienevorgaben erfordern auch weiterhin Flexibilität und Geduld in der Gestaltung des Unterrichts. Dabei hat sich vieles in Richtung Digitalisierung weiterentwickelt, das sich positiv auf die neurologische Lehre auswirken wird: Es mag sich lohnen, manche coronabedingten Veränderungen im Lehrplan auch in Zukunft beizubehalten.
Die Kommission Lehre der DGN sammelt innovative Konzepte, die sich möglicherweise auch langfristig als tragfähig erweisen. Bitte senden Sie Ihre Berichte und Vorschläge an annesophie.biesalski@klinikum-bochum.de.

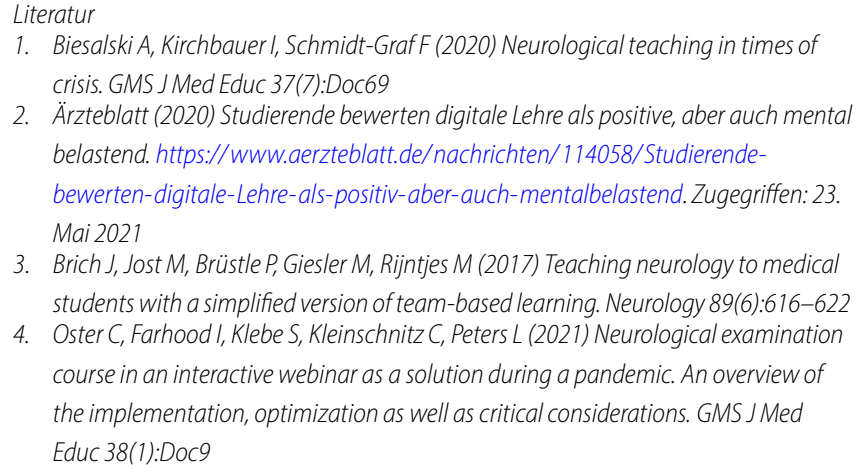

3. Brich J, Jost M, Brüstle P, Giesler M, Rijntjes M (2017) Teaching neurology to medical students with a simplified version of team-based learning. Neurology 89(6):616-622 4. Oster C, Farhood I, Klebe S, Kleinschnitz C, Peters L (2021) Neurological examination course in an interactive webinar as a solution during a pandemic. An overview of the implementation, optimization as well as critical considerations. GMS J Med Educ 38(1):Doc9

\section{Korrespondenzadresse}

\section{Dr. med. Anne-Sophie Biesalski}

Neurologische Klinik St. Josef Hospital Bochum Ruhr-Universität Bochum

Gudrunstraße 56, 44791 Bochum, Deutschland

anne-sophie.biesalski@klinikum-bochum.de

\section{Einhaltung ethischer Richtlinien}

Interessenkonflikt. A.-S. Biesalski, L. Peters, L. Tönges, S. Klebe, F. Fincke, K. Bornkamm, J. Brich und F. Schmidt-Graf geben an, dass kein Interessenkonflikt besteht.

Für diesen Beitrag wurden von den Autoren keine Studien an Menschen oder Tieren durchgeführt. Für die aufgeführten Studien gelten die jeweils dort angegebenen ethischen Richtlinien. 